\title{
New Insight of Discrete-Time Food Chain Interaction Model
}

\author{
Alaa Hussein Lafta \\ Science College / Mathematics Department \\ Baghdad University \\ Baghdad, Iraq \\ alaah74@yahoo.com
}

\author{
V. C. Borkar \\ Yeshwant Mahavidyalaya / Mathematics \& \\ Statistics Department \\ SRTM University \\ Nanded, India
}

\begin{abstract}
The discrete- time food chain interaction model is proposed. Steady state implies all possible equilibrium points. Stability conditions of arising equilibrium points are analyzed with numerical examples. Further, the dynamical behavior of the coexistence equilibrium point is obtained.
\end{abstract}

Keywords: discrete- time model, food chain, Stability theory, equilibrium point.

\section{INTRODUCTION}

Linear Lotka-Volterra model [1] consists of two interacting species vis. Prey and predator. Such mathematical models have long proved useful in describing how populations vary over time [2]. The other basic sequence of energy is the food chain which describes the movement from producer to consumer then to decomposer which called lowest-level, mid-level and top-level, respectively. The chain food is more important in understanding the food and energy relationship in the ecosystems.

The continuous time food chain of three or more species arise in (Hastings et al.1991, McCann et al.1994, Kuznetsov et al.1996, El-Owaidy 2001, and Chauvet 2002) while the Lotka-Volterra discrete-time food chain model proposed in ( Abd-Elalim et al. 2012, Hari 2014, and Sohel 2015).

In [10] we studied a non-linear discrete-time prey-predator model such that each population cans model by the logistic equation. For more insight and covering three species ecosystems, we extend our model in [10] by adding third population. The new model includes the lowest-level prey $X_{n}$ is preyed upon by a mid-level species $Y_{n}$, which, in turn, preyed upon by a top-level predator $Z_{n}$ as shown in the following system:

$$
\begin{aligned}
& X_{n+1}=X_{n}+h\left[a X_{n}\left(1-X_{n}\right)-b X_{n} Y_{n}\right] \\
& Y_{n+1}=Y_{n}+h\left[c Y_{n}\left(1-Y_{n}\right)+b X_{n} Y_{n}-f Y_{n} Z_{n}\right] \\
& Z_{n+1}=Z_{n}+h\left(-d+f Y_{n}\right) Z_{n}
\end{aligned}
$$

Where $a, b, c, d$ and $f$ are all positive parameters.

\section{STeady STATE}

In common parlance the system (1) has steady state if it is equal to the vector $X^{T}$ where $X=\{X, Y, Z\}$.Thus, this implies the following equilibrium points:

i. Fully washed out state

- $(x, y, z)=(0,0,0)$

ii- States in which only one of the three species is survives while the other two are not

- $(x, y, z)=(1,0,0)$

- $(x, y, z)=(0,1,0)$ 
iii- States in which only two of the three species are survives while the other one is not

- $(x, y, z)=\left(\frac{a c-b c}{a c+b^{2}}, \frac{a c+a b}{a c+b^{2}}, 0\right)$ where $a>b$.

- $\quad(x, y, z)=\left(0, \frac{d}{f}, \frac{c f-c d}{f^{2}}\right)$ where $f>d$.

iv- The co-existent steady states

$(x, y, z)=\left(\frac{a f-b d}{a f}, \frac{d}{f}, \frac{a c(f-d)+b(a f-b d)}{a f^{2}}\right)$ where $a \geq b$ and $f>d$.

\section{Stability AND Numerical ANALYSIS}

In general, if we want to study the stability of an equilibrium point in 3 dimensional systems we should firstly compute the Jacobian matrix and its characteristic equation then find out their roots as follows:

$$
\begin{gathered}
I(x, y, z)=\left[\begin{array}{lll}
a_{11} & a_{12} & a_{13} \\
a_{21} & a_{22} & a_{23} \\
a_{31} & a_{32} & a_{33}
\end{array}\right] \\
F(\lambda)=\lambda^{3}+M_{1} \lambda^{2}+M_{2} \lambda+M_{3}=0
\end{gathered}
$$

Where

$$
\begin{aligned}
& M_{1}=-\left(a_{11}+a_{22}+a_{33}\right) \\
& M_{2}=a_{11} a_{22}+a_{11} a_{33}+a_{22} a_{33}-a_{23} a_{32}-a_{12} a_{21}-a_{13} a_{31} \\
& M_{3}=a_{11}\left(a_{23} a_{32}-a_{22} a_{33}\right)+a_{21}\left(a_{12} a_{33}-a_{13} a_{32}\right)+a_{31}\left(a_{13} a_{22}-a_{12} a_{23}\right)
\end{aligned}
$$

Remark: The equilibrium point $(\mathrm{x}, \mathrm{y}, \mathrm{z})$ is stable if and only if all the absolute values of the roots of the equation (3) are less than one[10].

\subsection{Proposition}

The equilibrium points $(0,0,0)$ and $(1,0,0)$ in the states (i) and (ii), resp., are unstable.

\subsection{Proposition}

The equilibrium point $(0,1,0)$ is stable if $h<\min \left\{\frac{2}{b-a}, \frac{2}{c}, \frac{2}{d-f}\right\}$

\subsubsection{Example}

In the system (1) we have that the following parameters values

$a=0.12, b=0.15, c=0.1, d=0.95$ and $f=0.04$ where inertial point

$\left(x_{0}, y_{0}, z_{0}\right)=(0.5,0.3,0.4)$ and $h=0.1$. Under the above fixed values we see that the equilibrium point $(0,1,0)$ is stable. See the phase portrait and time series in the figure (1) bellow.

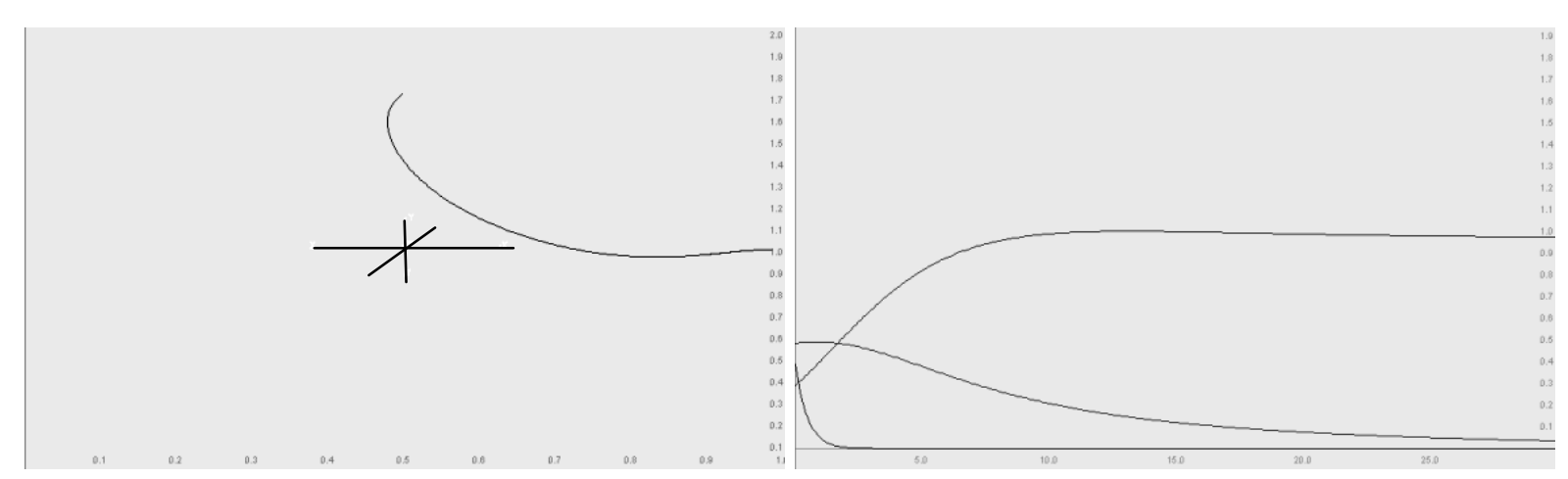

Figure (1). Stability of the equilibrium point $(0,1,0)$. 


\subsection{Proposition}

Let $a>b$ then we have that the equilibrium point $\left(\frac{a c-b c}{a c+b^{2}}, \frac{a c+a b}{a c+b^{2}}, 0\right)$ is stable if the following conditions hold:

1. $d-2<h<d$.

2. $\left|\frac{-T \pm \sqrt{T^{2}-4 D}}{2}\right|<1$

where

$$
T=-\left(2+h \frac{a c^{2}-a^{2} c}{a c+b^{2}}\right) \text { and } D=\frac{a c(1+h c-h a)+b^{2}}{a c+b^{2}}+h^{2} \frac{a^{2} b^{3} c-a b^{3} c^{2}-a b^{4} c+a b c^{3}+a^{3} b c^{2}-a^{3} c^{3}}{a c+b^{2}} .
$$

With the absence of top-level species, system (1) can be reduced to a sub system as discrete -time prey-predator which we studied before. This sub model exhibit complex dynamic behavior. For more details see [10].

\subsubsection{Example}

In the system (1) we have that the following parameters values $a=0.7, b=0.65, c=0.1, d=0.5 \quad$ and $f=1.14 \quad$ where inertial point $\left(x_{0}, y_{0}, z_{0}\right)=(0.5,0.3,0.4)$ and $h=0.1$. Under the above fixed values we see that the equilibrium point $(0.01015,1.06598,0)$ is stable. See the phase portrait and time series in the figure (2).
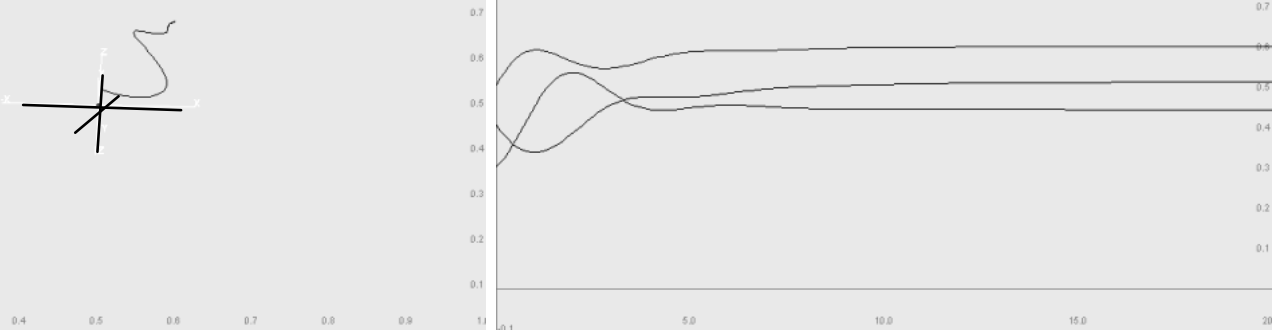

Figure (2). Stability of the equilibrium point $(0.01015,1.06598,0)$.

\subsection{Proposition}

If $f>d$ then the equilibrium point $\left(0, \frac{d}{f}, \frac{c f-c d}{f^{2}}\right)$ is stable if the following conditions hold:

1. $h<\frac{2 f}{b d-a f}$.

2. $\left|\frac{\frac{2 f-h c d}{f} \pm \sqrt{\left(\frac{2 f-h c d}{f}\right)^{2}-\frac{4(f-h c d(f-d))}{f}}}{2}\right|<1$

With the absence of lowest-level system (1) can be reduced to a sub system as discrete -time preypredator which studied by Liu and Xiao. This sub model has results reveal far richer dynamics of the discrete model compared with the continuous model. For more details see [12]

\subsubsection{Example}

In the system (1) we have that the following parameters values $a=0.1, b=0.65, c=5, d=0.5$ and $f=1.14 \quad$ where inertial point $\left(x_{0}, y_{0}, z_{0}\right)=(0.5,0.3,0.4)$ and $h=0.7$. Under the above fixed values we see that the equilibrium point $(0,0.4389,2.46229)$ is stable. See the bellow phase portrait and time series in the figure (3). 


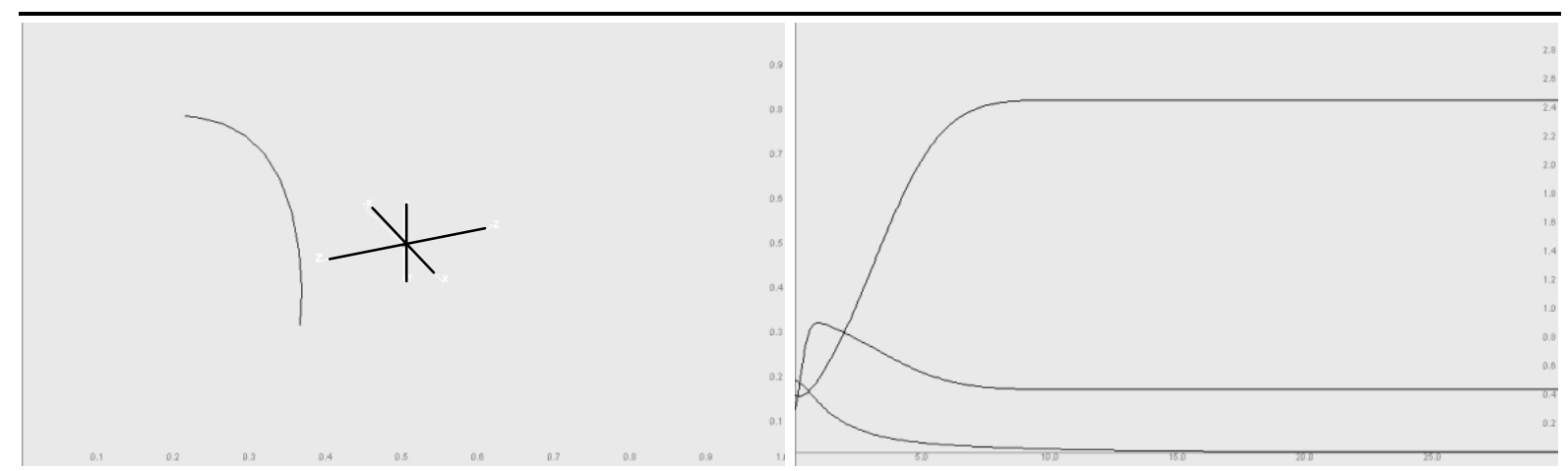

Figure (3). Stability of the equilibrium point(0,0.4389,2.46229).

\subsection{Proposition}

If $a \geq b$ and $f>d$ then the coexisting equilibrium point $\left(\frac{a f-b d}{a f}, \frac{d}{f}, \frac{a c(f-d)+b(a f-b d)}{a f^{2}}\right)$ is locally asymptotically stable if all the following Routh-Hurwitz conditions[11] hold:

$$
\begin{aligned}
& \text { 1. } 3+M_{1}-M_{2}-2 M_{3}>0 ; \\
& \text { 2. } 1-M_{2}+M_{3}\left(M_{1}-M_{3}\right)>0 ; \\
& \text { 3. } 1-M_{1}+M_{2}-M_{3}>0 .
\end{aligned}
$$

Where

$$
\begin{aligned}
& M_{1}=-\left(3+h \frac{f(1+c-a)+d(1+b-2 c)}{f}\right), \\
& M_{2}=\frac{3 f+2 h(b d+c f+d-a f-2 c d-f)}{f}+h^{2} \frac{a(b d-a f)(d+c f-2 c d-f)-(b d-a f)\left(b^{2} d+b d f\right)+a c d f(f-d)}{a f^{2}} \text { and } \\
& M_{3}=\frac{f+h(c f+d-f-2 c d-a f+b d)}{f}+h^{2} d \frac{a c f(f-d)+b^{2}(a f-b d)}{a f^{2}}+h^{3} d \frac{(b d-a f)[a c(f-d)+b(a f-b d)-f]}{a f^{2}}
\end{aligned}
$$

\subsubsection{Example}

In the figure (4), if the parameters value as follow $a=2.65, b=0.5, c=0.1, d=0.8$ and $f=0.9$ where inertial point $\left(x_{0}, y_{0}, z_{0}\right)=(0.5,0.5,0.5)$ and $h=0.87$ then the coexisting equilibrium point $(0.674151,0.888889,0.474726)$ in the system $(1)$ is stable Under the certain conditions. See the figure below:

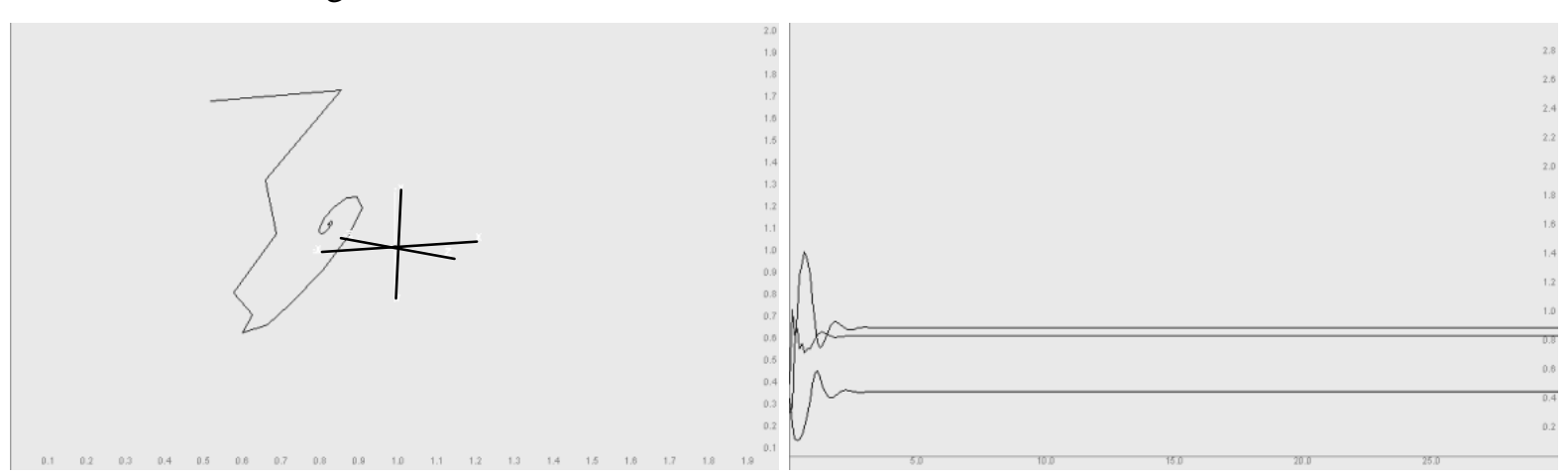

Figure (4). Stability of the equilibrium point (0.674151, 0.888889, 0.474726).

\section{Conclusion}

In our model, the lowest-level and mid-level are growth logistically with absence of other species. Also, if $a \geq b$ and $f>d$ then all possible equilibrium points are existing. With absence of the toplevel then the control parameter $h$ lays on the interval $[d-2, d]$ as a stability condition of the equilibrium point $\left(\frac{a c-b c}{a c+b^{2}}, \frac{a c+a b}{a c+b^{2}}, 0\right)$. 
The model of discrete-time food chain makes an interesting system in mathematical modeling as well as ecosystems science. It can be modified to model more complicated biological behavior. Finally, the phaser program is used to analysis our model numerically.

\section{ACKNOWLEDGEMENTS}

The authors are thankful the ICCR-India and the Baghdad University-Iraq for their financial support.

\section{REFERENCES}

[1] Boyce W. E. and Diprima R.C., Elementary differential equations and boundary value problems, $7^{\text {th }}$ ed., Wiley, (2001).

[2] Chauvet E., Paullet J.E., Previte J.P. and et.al. ,A Lotka-Volterra three-species food chain. Mathematics Magazine, 75, 243-255, (2002).

[3] Hastings A. and Powell T.,Chaos in three-species food chain. Ecology, 72, 896-903(1991).

[4] McCann K. and Yodzis P., Biological conditions for chaos in a three-species food chain. Ecology, 75,561-564, (1994).

[5] Kuznetsov Y.A. and Rinaldi S., Remarks on food chain dynamics. Mathematical Biosciences, 134, 1-33, (1996).

[6] El-Owaidy H.M., Ragab A.A. and Ismail M., Mathematical analysis of a food-web model. Applied Mathematics and Computation, 121(2), 3, 155-167, (2001).

[7] Elsadany A. A., Dynamical complexities in a discrete-time food chain. Computational Ecology and software, 2(2), 124-139, (2012).

[8] Hari B.P., A study on discrete model of three species syn-eco-systems with limited resources. IJMECS, 11, 38-44, (2014).

[9] Sohel S.M., Chaotic dynamics in discrete-time predator-prey food chain, Computational Ecology and software,5(1), 28-47,(2015) .

[10] Alaa H.L. and Borkar V. C., Flip bifurcation and chaos control in discrete-time Prey-predator model, IRJES, 4(7), 44-50, (2015).

[11] Murray J. D., Mathematical biology, $3^{\text {rd }}$ edition, Springer-Verlage, Berlin, (2004).

[12] Liu X. and Xiao D., Complex dynamic behaviors of a discrete-time predator-prey system, Chaos Solitons Fractals 32, 80-94, (2007).

\section{AUTHORS' BIOGRAPHY}

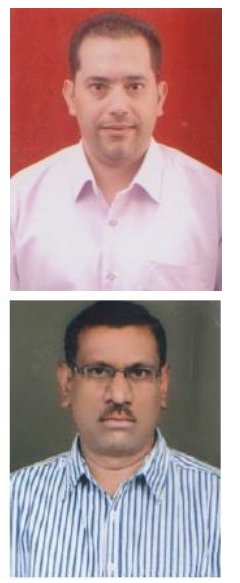

Alaa Hussein Lafta, is working as an assistant professor at the Mathematics department, Science College, Baghdad University, Baghdad-Iraq. He is a research student working in the Swami Ramanand Teerth Marathwada University, Nanded. His area of research is dynamical systems and it's applications in various fields.

Dr. V. C. Borkar, working as Associate Professor and Head in Department of Mathematics and Statistics Yeshwant Mahavidyalaya Nanded, Under Swami Ramanand Teerth Marathwada University, Nanded (M.S) India. His area of specialization is functional Analysis. He has near about 16 years research experiences. He completed one research project on Dynamical system and their applications. The project was sponsored by UGC, New Delhi. 